\title{
Effect of the intermittent Pringle maneuver on liver damage after hepatectomy: a retrospective cohort study
}

\author{
Xiaolin Wei', Wenjing Zheng ${ }^{1}$, Zhiqing Yang ${ }^{2}$, Hui Liu', Tengqian Tang ${ }^{2}$, Xiaowu Li ${ }^{1 *}$ (D) and Xiangde Liu ${ }^{2 *}$
}

\begin{abstract}
Background: The Pringle maneuver (PM) interrupts the blood flow through the hepatic artery and portal vein to help control bleeding. This study analyzes the effects of the intermittent Pringle maneuver (IPM) on the surgical process and postoperative liver injury.
\end{abstract}

Methods: This study retrospectively evaluated 182 hepatocellular carcinoma patients who underwent hepatectomy. In the IPM group, hepatic blood flow was intermittently interrupted via clamping, with cycles of 10 minutes of inflow occlusion followed by 5 minutes of reperfusion that were repeated until the end of the surgery. In the nonIPM group, liver resection was performed without hepatic vascular blockage.

Results: For postoperative complications, the incidence rates of ascites and pleural effusion in the IPM group were significantly lower than those in the non-IPM group. The postoperative hospitalization time in the IPM group was significantly lower than that in the non-IPM group ( $p=0.0008)$. On the first day after the operation, the platelet count was significantly lower $(p=0.0381)$ but the prothrombin time (PT) $(p=0.0195)$ and activated partial thromboplastin time (APTT) ( $p=0.0071)$ were significantly higher in the non-IPM group than those in the IPM group. At discharge, only albumin was significantly higher in the non-IPM group than that in the IPM group $(p=0.0303$ ). Regression analysis showed that a prolonged interruption time was related to increased ALT and AST levels on the first day after surgery, but not on the seventh day or at discharge.

Conclusion: The IPM does not cause additional liver damage during hepatectomy, and use of the IPM results in shorter hospital stays compared to surgery without using the IPM. The results of this study require further confirmation because of the retrospective design.

Keywords: Hepatectomy, Pringle maneuver, Liver damage, Retrospective.

\section{Introduction}

Hepatectomy is the most effective method to treat hepatobiliary cancer, such as hepatic carcinoma. Massive bleeding is usually the major problem in hepatectomy. Although successful hepatectomy does not necessarily require blocking hepatic blood flow [1], controlling the hepatic blood flow is helpful for maintaining a relatively bloodless surgical environment, disconnecting the liver,

\footnotetext{
* Correspondence: lixiaowu8055@yeah.net; liuxiangde07@outlook.com ${ }^{1}$ Shenzhen University General Hospital \& Shenzhen University Clinical Medical Academy, No. 1098, Xue Yuan Avenue, Xili University Town, Shenzhen 518055, Guangdong, China

${ }^{2}$ Southwest Hospital, Third Military Medical University (Army Medical University), No. 30, Gaotanyan Street, Shapingba District, Chongqing 400038, China
}

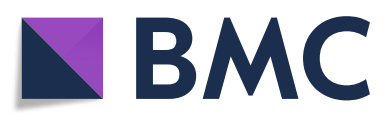

(c) The Author(s). 2019 Open Access This article is distributed under the terms of the Creative Commons Attribution 4.0 International License (http://creativecommons.org/licenses/by/4.0/), which permits unrestricted use, distribution, and reproduction in any medium, provided you give appropriate credit to the original author(s) and the source, provide a link to the Creative Commons license, and indicate if changes were made. The Creative Commons Public Domain Dedication waiver (http://creativecommons.org/publicdomain/zero/1.0/) applies to the data made available in this article, unless otherwise stated. reducing intraoperative bleeding, and shortening the operation time. The Pringle maneuver (PM) is a surgical maneuver used to interrupt the blood flow through the hepatic artery and portal vein to help control bleeding from the liver; the PM is technically easy to implement and frequently used by surgeons [2].

However, the advantages and disadvantages of the PM remain controversial $[3,4]$. Unlike the effect of the PM on liver dysfunction in animal experiments, in clinical practice, although blocking hepatic blood flow leads to hepatic ischemia, metabolism in the human liver is not significantly affected $[5,6]$. The main reason may be the more abundant collateral circulation in the human liver than that in the livers of animal models. In addition, the 
tolerance of the liver to warm ischemia and ischemia-reperfusion injury induced by the PM may be related to the duration of hepatic ischemia [7]. An intermittent PM (IPM) can partially reduce ischemic damage to the residual liver, thus prolonging the total tolerable time of the residual liver to ischemia.

The ability of the residual liver to regenerate is another important aspect of evaluating the success of hepatectomy, and the effect of intraoperative hepatic blood flow occlusion on liver regeneration remains controversial [8]. Thermal ischemia of the liver may lead to protein synthesis dysfunction in hepatocytes. However, a study has shown that the PM does not affect liver regeneration after hepatectomy, and short-term thermal ischemia can even accelerate liver regeneration [9]. In this study, we retrospectively analyzed the effects of the IPM on the surgical process and recovery from postoperative liver injury and compared hepatectomy with the IPM to hepatectomy without the IPM.

\section{Patients and methods Patients}

This study retrospectively evaluated 182 hepatocellular carcinoma patients who underwent open hepatectomy in the hepatological surgery department of our hospital from 2012 to 2016. The patient age range was 21 to 84 years old, with 150 males and 32 females. In total, 108 patients were included in the IPM group, and 74 patients were included in the non-IPM group. The inclusion criteria were as follows: age $\geq 18$ years and hepatocellular carcinoma requiring hepatectomy. The following patients were excluded from this study: patients who previously underwent major operations on the liver or adjacent areas and patients who did not undergo liver resection. All included patients were consecutive patients who met the inclusion criteria. This study was performed in accordance with the Declaration of Helsinki and was approved by the Ethics Committee of Southwest Hospital, Third Military Medical University (Army Medical University).

\section{Preoperative evaluation}

The gender, age, and clinical diagnosis of each patient were recorded before the operation. Liver-related complications and other comorbidities were also recorded. Preoperative laboratory blood tests included alanine aminotransferase (ALT), aspartate aminotransferase (AST), serum albumin, total bilirubin, hepatitis B surface antigen (HBsAg), and alpha-fetoprotein (AFP) levels, the presence of hepatitis B virus (HBV) deoxyribonucleic acid (DNA), the platelet count, and the prothrombin time (PT). The Child-Pugh classification scheme was used to assess the liver reserve function of the patients [10]. For patients with hepatic carcinoma, tumor, node, metastasis (TNM) staging was evaluated.

\section{Surgical procedure}

All surgical procedures were performed by our departmental doctors to ensure consistency. The extent of liver resection was determined by precise segmental resection. The hepatoduodenal ligament was clamped to control the hepatic vasculature until the hepatic artery pulse disappeared distally. In the IPM group, the hepatic vasculature was intermittently clamped, with cycles of $10 \mathrm{~min}$ of inflow occlusion followed by $5 \mathrm{~min}$ of reperfusion that were repeated until the end of the surgery (additional illustrations and movie files show this in more detail see Additional file 1 and Additional file 2). In the non-IPM group, liver resection was performed without hepatic blockage. The duration of hepatic vascular occlusion (excluding the open period), the number of occlusions, the duration of the operation, and the amount of bleeding during the operation were recorded.

\section{Postoperative management}

Postoperative complications and the durations of hospital and intensive care unit (ICU) stays were recorded. The leukocyte count, neutrophil ratio, platelet count, ALT, AST, serum albumin, total bilirubin, and D-dimer (D-D) levels, PT, and activated partial thromboplastin time (APTT) were measured on the first, third, fifth, and seventh postoperative days and at discharge. No results were recorded when the test was normal or the patient refused the test.

\section{Statistical analysis}

The qualitative data are expressed as frequencies (percentages), and statistical significance was evaluated using the $x^{2}$ test. Quantitative data are expressed as the mean \pm standard deviation, and the groups were compared using analysis of variance (ANOVA) if the data were normally distributed as detected by skewness and kurtosis tests. Non-normally distributed data were compared with the Kruskal-Wallis test and are expressed as the medians and quartiles. The $p$ value in multiple analyses was corrected by false discovery rate (FDR) methods. Linear regression analysis was used to analyze the interruption time and postoperative liver injury. Recovery of liver function with increasing postoperative time in IPM patients and non-IPM patients was further analyzed. This seemingly unrelated estimation was used to test the difference in regression equation coefficients [11]. All the calculations were performed with STATA 14.0 software (StataCorp LLC, TX, US), and results with $p<0.05$ were considered significant. 


\section{Results}

No difference in the mean age or sex ratio was found between the IPM and non-IPM groups. No significant differences were identified between the two groups in terms of hepatic comorbidities. No significant differences in the rates of hypertension and diabetes were noted between the two groups. With regard to the laboratory tests, no significant differences in the ALT, AST, albumin, total bilirubin, HBsAg, or AFP levels, PT, platelet count, or amount of HBV DNA were found between the two groups (Table 1).

The operative time and bleeding volume in the IPM group were not significantly different from those in the

Table 1 Characteristic of included patients

\begin{tabular}{|c|c|c|c|}
\hline & Non-IPM & IPM & $p$ value \\
\hline$n$ & 74 & 108 & \\
\hline Age (year) & $49.15 \pm 11.10$ & $51.45 \pm 12.45$ & 0.202 \\
\hline Sex (male/female) & $63 / 11$ & $87 / 21$ & 0.425 \\
\hline \multicolumn{4}{|l|}{ Liver-related basic disease } \\
\hline Cirrhosis & 26 & 40 & 0.793 \\
\hline Portal hypertension & 10 & 13 & 0.768 \\
\hline Hypersplenism & 11 & 14 & 0.714 \\
\hline $\mathrm{HbsAg}(+)$ & 64 & 83 & 0.105 \\
\hline HBV DNA(+) & 48 & 52 & 0.07 \\
\hline AFP $>40$ & 36 & 55 & 0.926 \\
\hline Others & 16 & 17 & 0.312 \\
\hline \multicolumn{4}{|l|}{ Non-liver combined disease } \\
\hline Hypertension & 6 & 5 & 0.333 \\
\hline Diabetes & 8 & 14 & 0.662 \\
\hline Others & 14 & 16 & 0.464 \\
\hline \multicolumn{4}{|l|}{ Liver injury } \\
\hline $\mathrm{ALT}(\mathrm{U} / \mathrm{L})$ & $40(24-75)$ & $36.6(22-64)$ & 0.5674 \\
\hline AST (U/L) & $44(29-70)$ & $45(30.6-75)$ & 0.9066 \\
\hline \multicolumn{4}{|l|}{ Liver function reserve } \\
\hline Albumin (g/L) & $41.4(38.6-44.8)$ & $41.9(38.1-45.2)$ & 0.944 \\
\hline Total bilirubin $(\mu \mathrm{mol} / \mathrm{L})$ & $16.5(13.3-20.4)$ & $15.8(12.75-22.5)$ & 0.9965 \\
\hline \multicolumn{4}{|l|}{ Child-Pugh classification } \\
\hline 5 & 60 & 91 & \\
\hline 6 & 5 & 4 & \\
\hline 7 & 4 & 6 & \\
\hline 8 & 2 & 5 & 0.721 \\
\hline \multicolumn{4}{|l|}{ Coagulation function } \\
\hline Prothrombin time (seconds) & $11.60(11.1-12.3)$ & $11.7(11-12.4)$ & 0.6764 \\
\hline Platelet $\left(\times 10^{9} / \mathrm{L}\right)$ & 165 (123-202) & $185(128-248)$ & 0.0683 \\
\hline
\end{tabular}

Characteristics of hepatic carcinoma patients

TNM stage

$\begin{array}{llll}1 & 42 & 63 & 18 \\ 2 & 13 & 20 & 0.954 \\ 3 & 12 & 6 & 0.551 \\ 4 & 6 & 25 & 0.904 \\ \text { Cancer embolus } & 20 & 6 & 0 \\ \text { Lymphatic metastasis } & 3 & 25\end{array}$

Abbreviations: AFP alpha-fetoprotein, ALT alanine transaminase, AST aspartate aminotransferase, IPM intermittent Pringle maneuver, TNM stage tumor, lymph node, metastasis stage 
non-IPM group. No statistical differences in the extent of hepatectomy and the number of injured hepatic segments were found between the two groups. The duration of portal occlusion was $50(30-80) \mathrm{min}$, and the number of occlusions was 5 (3-8) in the IPM group. No differences were found between the two groups in terms of the performance of cholecystectomy $(p=0.075)$ and other accessory procedures $(p=0.312)$ (Table 2$)$.

In terms of postoperative complications, no significant differences in postoperative bleeding, bile leakage, incision infection, abdominal abscess, incision effusion, pulmonary infection, hepatic insufficiency/liver failure, or death were found between the two groups. The incidence rates of ascites $(p=0.024)$ and pleural effusion $(p=0.035)$ in the IPM group were significantly lower than those in the nonIPM group. The postoperative hospitalization time in the IPM group was significantly shorter than that in the nonIPM group $(p=0.0008)$ (Table 2$)$. Two patients died in the IPM group; the causes of death were organ failure after abdominal infection and cardiac arrest due to cardiac insufficiency. However, no difference in the incidence of abdominal infection or incision infection was found between the two groups.

On the first day after the operation, the leukocyte count, neutrophil ratio, and ALT, AST, albumin, total bilirubin, and D-D levels were not significantly different

Table 2 Characteristic of patients during and after surgery

\begin{tabular}{|c|c|c|c|}
\hline & Non IPM & IPM & $p$ value \\
\hline Surgery time (min) & $300(245-390)$ & $288.5(219-356)$ & 0.187 \\
\hline Amount of bleeding (ml) & $500(300-800)$ & $400(200-800)$ & 0.0941 \\
\hline Hepatic portal occlusion time (min) & 0 & $50(30-80)$ & NA \\
\hline Count of occlusion & 0 & $5(3-8)$ & NA \\
\hline \multicolumn{4}{|l|}{ Type of operation } \\
\hline Partial lobectomy & 44 & 59 & \\
\hline Left lobectomy & 9 & 16 & \\
\hline Right lobectomy & 18 & 28 & \\
\hline Trisegmentectomy & 3 & 5 & 0.922 \\
\hline \multicolumn{4}{|l|}{ Number of injured hepatic segments } \\
\hline 1 & 6 & 17 & \\
\hline 2 & 27 & 27 & \\
\hline 3 & 16 & 27 & \\
\hline 4 & 22 & 31 & \\
\hline 5 & 3 & 6 & 0.354 \\
\hline \multicolumn{4}{|l|}{ Attach surgery } \\
\hline Cholecystectomy & 54 & 65 & 0.075 \\
\hline Others & 16 & 17 & 0.312 \\
\hline \multicolumn{4}{|l|}{ Postoperative complication } \\
\hline Bleeding & 0 & 2 & 0.239 \\
\hline Biliary fistula & 2 & 0 & 0.086 \\
\hline Incision infection & 1 & 1 & 0.787 \\
\hline Intra-abdominal abscess & 1 & 2 & 0.794 \\
\hline Sectional effusion & 4 & 9 & 0.451 \\
\hline Ascites & 22 & 17 & 0.024 \\
\hline Pulmonary infection & 27 & 27 & 0.096 \\
\hline Pleural effusion & 48 & 53 & 0.035 \\
\hline Respiratory failure & 2 & 0 & 0.086 \\
\hline Liver failure/dysfunction & 0 & 0 & NA \\
\hline Death & 0 & 2 & 0.239 \\
\hline Hospital duration (day) & $15(12-17)$ & $12(10-16)$ & 0.0008 \\
\hline ICU duration (day) & $3(2-3)$ & $2(2-3)$ & 0.1478 \\
\hline
\end{tabular}


Table 3 Results of blood biochemistry and coagulation function after operation

Non IPM IPM $\quad p$ value

First day after operation

$\begin{array}{llll}\text { WBC } & 11.38(9.05-14.08) & 12.15(9.85-15.3) & 0.2386 \\ \text { NLR } & 88.08 \pm 3.68 & 87.33 \pm 4.12 & 0.2307 \\ \text { PLT } & 132(105-168) & 152(114-210) & 0.0381 \\ \text { ALT } & 242.5(129-336) & 157.1(98.2-384.3) & 0.1709 \\ \text { AST } & 254(171-418) & 208.8(126.05-400.55) & 0.1148 \\ \text { ALB } & 32.48 \pm 5.11 & 32.24 \pm 6.05 & 0.7739 \\ \text { TB } & 25.8(17.5-40.9) & 22.4(15.7-33) & 0.07 \\ \text { PT } & 15.3(14.3-17) & 14.3(13.5-15.8) & 0.0195 \\ \text { APTT } & 36.3(32-43.4) & 33.2(28.6-38.7) & 0.0071 \\ \text { D-Dimer } & 2.92(0.83-4.46) & 3.765(2.06-4.85) & 0.1478\end{array}$

Third day after operation

$\begin{array}{ll}\text { WBC } & 8.43(5.78-10.59) \\ \text { NLR } & 83.51 \pm 5.3 \\ \text { PLT } & 106.5(74-158) \\ \text { ALT } & 133.9(92-229.8) \\ \text { AST } & 86(67-132) \\ \text { ALB } & 37.84 \pm 5.09 \\ \text { TB } & 22.7(15.5-37.5) \\ \text { PT } & 15.8 \pm 3 \\ \text { APTT } & 40.8(33.4-52.4) \\ \text { D-Dimer } & 3.84(0.893-6.38)\end{array}$

Fifth day after operation

$\begin{array}{ll}\text { WBC } & 7.89 \pm 3.05 \\ \text { NLR } & 75.37 \pm 8.86 \\ \text { PLT } & 110.5(72-176) \\ \text { ALT } & 74.5(53-104) \\ \text { AST } & 50(35-62) \\ \text { ALB } & 36.2 \pm 4.35 \\ \text { TB } & 24.05(13.7-32.8) \\ \text { PT } & 15.8(14.05-17.65) \\ \text { APTT } & 40.85(34.35-48.95) \\ \text { D-Dimer } & 5.5(1.15-9.53)\end{array}$

$8.39(6.47-10.84)$

0.7004

$80.5 \pm 5.91$

$126(100-167)$

$104.1(71-245)$

89 (64-140.4)

$37.08 \pm 5.43$

$26.05(17.4-34.7)$

$14.9 \pm 2.4$

$38.15(32.6-42.9)$

$4.65(2.8-9.1)$

0.0057

0.075

0.2797

0.7739

0.4332

0.6339

0.2071

0.1275

0.1136

0.8301

0.2143

0.0739

0.3716

0.5623

0.2176

0.807

0.2038

0.0526

0.3496

Seventh day after operation

$\begin{array}{llll}\text { WBC } & 7.69(7.08-11.19) & 8.28(7.39-12.04) & 0.529 \\ \text { NLR } & 70.89 \pm 7.01 & 69.82 \pm 8.18 & 0.6239 \\ \text { PLT } & 178.11 \pm 56.26 & 192.69 \pm 98.42 & 0.5614 \\ \text { ALT } & 49(44-80) & 55(42-100) & 0.5803 \\ \text { AST } & 32(23-49) & 38.1(29-53.9) & 0.2185 \\ \text { ALB } & 36.23 \pm 4.37 & 35.91 \pm 3.52 & 0.7621 \\ \text { TB } & 19.5(15.1-51.7) & 21.1(16.1-32.3) & 0.9008 \\ \text { PT } & 14.45(13.6-17.25) & 15(13.6-16.6) & 0.9422 \\ \text { APTT } & 35.8(29.7-46.85) & 32.2(29.7-61) & 0.8283\end{array}$

Table 3 Results of blood biochemistry and coagulation function after operation (Continued)

\begin{tabular}{|c|c|c|c|}
\hline & Non IPM & IPM & $p$ value \\
\hline D-Dimer & $5.715(1.42-13.31)$ & $7.41(0.748-10.93)$ & 0.665 \\
\hline \multicolumn{4}{|c|}{ Discharge from hospital } \\
\hline WBC & $6.35 \pm 2.66$ & $6.49 \pm 2.27$ & 0.7368 \\
\hline NLR & $67.56 \pm 9.15$ & $67.09 \pm 7.5$ & 0.7466 \\
\hline PLT & $184.5(128-249)$ & $178(126-223)$ & 0.5397 \\
\hline ALT & $46(35-61)$ & $48.8(32.1-65.9)$ & 0.2943 \\
\hline AST & $35(25-46)$ & $36.6(28.1-51)$ & 0.2819 \\
\hline ALB & $37.5(33-40.8)$ & $35.5(31.9-37.9)$ & 0.0303 \\
\hline TB & $16.4(13-25.5)$ & 16.7(11.9-25.2) & 0.8516 \\
\hline PT & $13.05(12.2-14.9)$ & $13.1(12.2-14.1)$ & 0.9695 \\
\hline APTT & $28.8(27.4-34.8)$ & $30.45(28.5-35)$ & 0.3497 \\
\hline D-Dimer & $8.67 \pm 5.91$ & $9.54 \pm 5.14$ & 0.7053 \\
\hline
\end{tabular}

Abbreviations: WBC white blood cell, NLR neutrophil to lymphocyte ratio, $P L T$ platelet, $A L T$ alanine transaminase, $A S T$ aspartate transaminase, $A L B$ albumin, $T B$ total bilirubin, $P T$ prothrombin time, APTT activated partial thrombin time Footnote: Normal distribution quantitative data is expressed as mean \pm standard deviation and compare the group comparison using analysis of variance; non-distribution data is expressed as median and quartile and compare the group comparison using Kruskal-Wallis test

between the two groups. The platelet count in the nonIPM group was significantly lower than that in the IPM group $(p=0.0381)$, but the PT $(p=0.0195)$ and APTT $(p=0.0071)$ in the non-IPM group were significantly higher than those in the IPM group. On the third day after the operation, only the neutrophil ratio remained significantly higher in the non-IPM group than that in the IPM group $(p=0.0057)$, and no significant differences were found in the other indexes. On the fifth and seventh postoperative days, no significant differences in any of the indexes were observed between the two groups. At discharge, only albumin in the non-IPM group was significantly higher than that in the IPM group $(p=0.0303)$ (Table 3$)$. The statistically different results did not change after correction by the FDR method. The length of hospitalization showed the most pronounced difference between the non-IPM and IPM groups $(p=0.0008)$, followed by the third-day neutral cell ratio $(p=0.0057)$ and the first-day APTT $(p=0.0071)$. Importantly, no significant differences in ALT and AST levels were noted between the two groups.

The effects of hepatic interruption time on the levels of ALT and AST after surgery were analyzed by regression (Table 4). Univariate regression showed that a prolonged interruption time was related to increased levels of ALT (coef $=1.66, p=0.001)$ and AST $(\operatorname{coef}=2.00$, $p=0.002$ ) on the first day after the operation. On the fifth day after the operation, the interruption time was significantly correlated with the level of ALT (coef = $0.41, p=0.038)$. No correlation was found between the 
Table 4 Univariate and multivariate analysis of hepatic vascular occlusion time and postoperative ALT AST results

\begin{tabular}{|c|c|c|c|c|c|}
\hline \multicolumn{2}{|c|}{ Hepatocellular carcinoma patients } & \multicolumn{2}{|l|}{ Univariate analysis } & \multicolumn{2}{|l|}{ Multivariate analysis\# } \\
\hline After operation & Index & Coef. $(95 \% \mathrm{Cl})$ & $p$ value & Coef. $(95 \% \mathrm{Cl})$ & $p$ \\
\hline \multirow[t]{2}{*}{ 1st day } & $\mathrm{ALT}$ & $1.66(0.67,2.65)$ & 0.001 & $1.61(0.6,2.63)$ & 0.002 \\
\hline & AST & $2(0.76,3.24)$ & 0.002 & $1.8(0.5,3.1)$ & 0.007 \\
\hline \multirow[t]{2}{*}{ 3rd day } & $\mathrm{ALT}$ & $0.36(-0.37,1.09)$ & 0.332 & $0.42(-0.34,1.18)$ & 0.277 \\
\hline & AST & $0.24(-0.06,0.54)$ & 0.118 & $0.26(-0.04,0.55)$ & 0.088 \\
\hline \multirow[t]{2}{*}{ 5th day } & ALT & $0.41(0.02,0.8)$ & 0.038 & $0.41(0.01,0.81)$ & 0.043 \\
\hline & AST & NA & & $0.11(-0.04,0.26)$ & 0.140 \\
\hline \multirow[t]{2}{*}{ 7th day } & ALT & NA & & NA & NA \\
\hline & AST & NA & & NA & NA \\
\hline \multirow[t]{2}{*}{ Discharge } & ALT & $0.08(-0.08,0.24)$ & 0.315 & $0.1(-0.06,0.26)$ & 0.213 \\
\hline & AST & $-0.01(-0.13,0.1)$ & 0.817 & $-0.04(-0.17,0.08)$ & 0.526 \\
\hline
\end{tabular}

\#Adjust for age, sex, and preoperative ALT/AST variants

interruption time and the levels of ALT and AST on the seventh day after the operation or at discharge. Multivariate analysis yielded similar results.

Regression analysis was used to analyze the relationships between the levels of ALT and AST and postoperative hospitalization time in the IPM group and non-IPM group. The regression relationships between the levels of ALT $(y=-14.44 x+301.35, p<0.001)$ and AST $(y=-17.88 x+$ 350.36, $p<0.001)$ and postoperative time were significant in the non-IPM group (Fig. 1a, c). The regression relationships between the levels of ALT $(y=-14.21 x+272.46$, $p<0.001)$ and AST $(y=-18.41 x+322.44, p<0.001)$ and postoperative time were also significant in the IPM group (Fig. 1b, d). No significant difference in the regression coefficients was found between the two groups (ALT: $p=$ 0.9387; AST: $p=0.8901$ ).

\section{Discussion}

This study retrospectively analyzed 182 hepatocellular carcinoma patients who underwent hepatectomy and evaluated the effect of the IPM on the postoperative hepatocellular injury. In our study, the incidence rates of pleural effusion and ascites were higher in the non-IPM group than those in the IPM group. The hospitalization time in the IPM group was clearly shorter than that in the non-IPM group. The platelet count in the non-IPM group was significantly lower than that in the IPM group. The PT and APTT in the non-IPM group were significantly higher than those in the IPM group on the first day after surgery. On the third day after the operation, the neutrophil ratio in the non-IPM group was significantly higher than that in the IPM group. At discharge, only albumin in the non-IPM group was significantly higher than that in the IPM group. Other indicators showed no significant differences between the two groups. In the regression analysis of the levels of ALT and AST and the total hepatic interruption time, the ALT and AST levels on the first day after surgery increased with prolongation of the interruption time.

In this study, we found that the PT and APTT in the non-IPM group were significantly higher than those in the IPM group, and the platelet count was lower in the non-IPM group. More blood loss was recorded in the non-IPM group than that in the IPM group, but the difference was not statistically significant $(p=0.0941)$, which may explain the increased PT and APTT in the non-IPM group. The difference disappeared on the third day after surgery. A prospective study also suggested that the PM can reduce bleeding during hepatectomy, minimize hemodynamic disturbances, and protect liver function in the early postoperative period [12]. The PM is even considered safe for patients with severe cirrhosis [6]. In addition, intermittent occlusion of the hepatic hilum may result in hepatic tissue tolerance of and protection against ischemia-reperfusion injury [7]. Liver ischemia preconditioning before the PM is applied has also been shown to enhance liver tolerance [13]. In liver transplantation, intermittent blood flow interruption has no significant effect on liver function and injury [14, 15]. Our study also indicated that hepatic vascular occlusion had no significant effect on liver injury and liver function.

This study compared hepatectomies with and without the IPM. Meanwhile, controversy remains regarding the use of continuous and intermittent PMs. A study suggested that continuous PMs can more successfully reduce liver injury and promote liver recovery than IPMs [16]. However, another study reported no significant difference in liver injury between patients undergoing continuous and intermittent interruption of the hepatic blood flow [17]. This finding may be related to the duration of the interruption time. If the duration of a single interruption event does not exceed the threshold for liver ischemia-reperfusion injury, then liver damage will not occur. Once the threshold is exceeded, however, the blood flow interruption will cause 


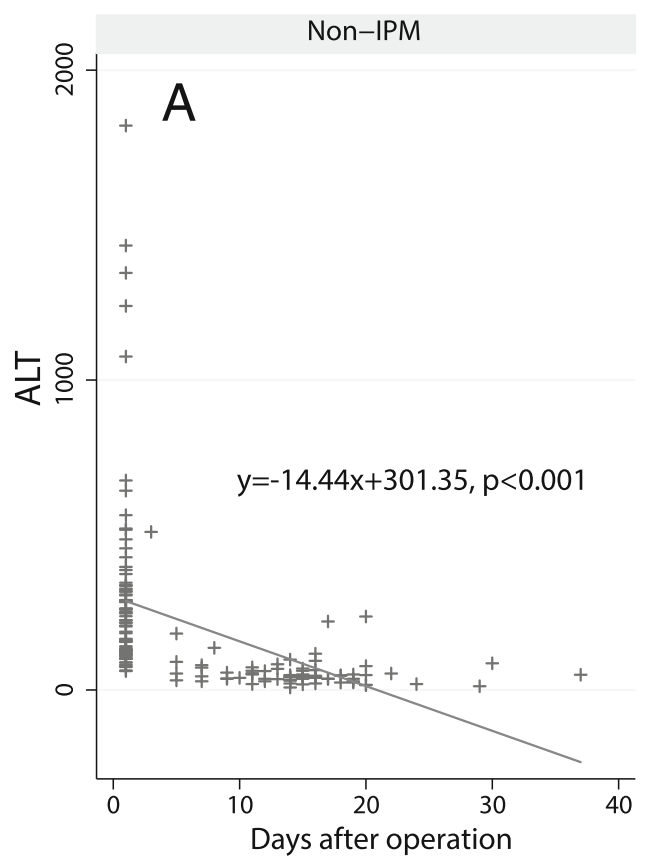

IPM
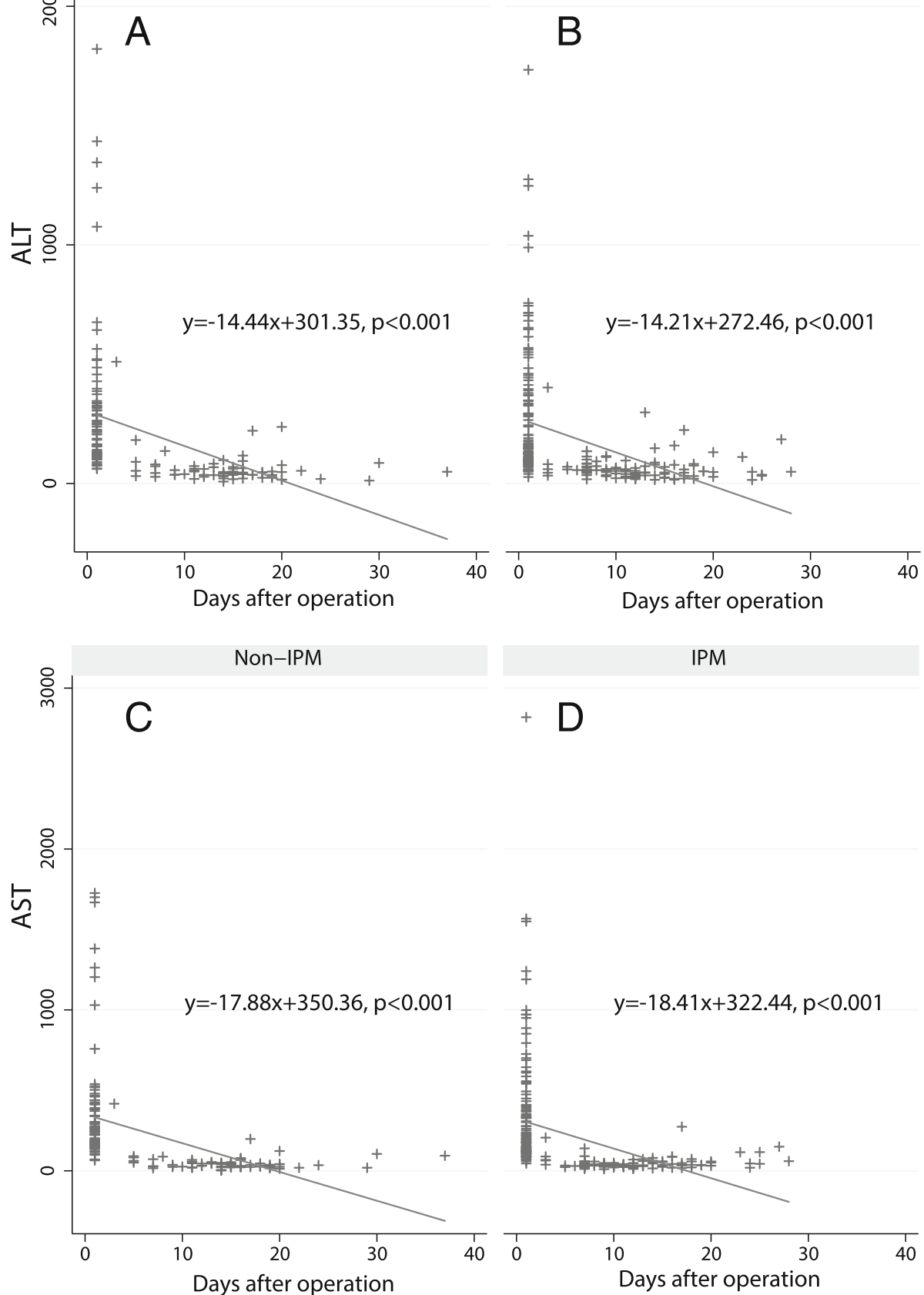

Fig. 1 Regression analysis of ALT/AST recovery with postoperative time in non-IPM and IPM patients. a ALT changes in non-IPM patients. b ALT changes in IPM patients. c AST changes in non-IPM patients. d AST changes in IPM patients

liver damage. Therefore, the effect of the duration of a single interruption event on postoperative liver recovery may exceed that of the total interruption time.

In a clinical prospective randomized controlled trial (RCT), the performance of the IPM with intervals of 30 min was considered safe [18]. In other retrospective clinical studies, the authors concluded that the IPM with clamping times exceeding 60-120 min was still safe [19-21]. In this study, more intensive circulation was achieved using an intermittent strategy with cycles of $10 \mathrm{~min}$ of inflow occlusion followed by $5 \mathrm{~min}$ of reperfusion. This intermittent strategy did not cause significant liver damage in this study. Therefore, the interruption strategy should be clearly stated in future reports on the IPM to allow comparisons among studies. 
Regarding recovery from hepatocellular injury after surgery, the levels of ALT and AST decreased gradually as the postoperative time increased in both the IPM and non-IPM groups, and no significant difference in postoperative liver injury was noted between the two groups in this study. In terms of postoperative complications, this study found higher incidence rates of ascites and pleural effusion in the non-IPM group than those in the IPM group. In an RCT with patients with liver tumors, the population receiving the IPM had higher rates of subclinical ascites and pleural effusion than the nonIPM population [22] in contrast to our reported results; however, the ascites and pleural effusion rates were determined based on radiological measurements rather than clinical testing. In addition, this study also concluded that the IPM had no significant effect on the length of hospitalization after surgery [22].

A recent retrospective study analyzed hepatectomy patients in the liver-targeted National Surgical Quality Improvement Program (NSQIP) database (2014-2016), but the heterogeneity of this study was relatively high [23]. The study indicated that the PM was associated with a longer total hospital length of stay based on a comparison with non-PM cases. Other studies have reported no difference in the incidence of post-hepatectomy liver failure or the need for blood transfusion. Our study further defined IPM strategies and hepatocellular carcinoma patients undergoing hepatectomy and revealed that the IPM does not cause additional liver damage. No significant difference in intraoperative blood loss was observed between the study groups. We believe that the IPM can facilitate better surgical visualization and concluded that application of the PM results in a shorter hospitalization time based on this study, but this outcome may be related to the characteristics of various patient populations or even to the policies of local hospitals.

Finally, whether ischemia-reperfusion injury caused by the PM can promote the recurrence and metastasis of hepatic tumors and affect patient prognosis remains controversial in the clinical setting. Research results suggest that the IPM is safe for patients with liver cancer [24-26]. This study mainly analyzed the role of the IPM in liver damage and liver injury after hepatic surgery, and long-term follow-up results are required to determine the impact of the IPM on liver cancer patients.

\section{Conclusions}

This study concluded that the IPM does not cause additional liver damage during hepatectomy. In addition, the use of the IPM results in shorter hospital stays compared to surgery without using the IPM. However, the results of this study require further confirmation because of the retrospective design.

\section{Additional files}

Additional file 1: Illustration of surgical procedure. (DOCX $3149 \mathrm{~kb}$ )

Additional file 2: Video of surgical procedure. (FLV $15850 \mathrm{~kb}$ )

\section{Abbreviations}

AFP: Alpha-fetoprotein; ALT: Alanine aminotransferase; ANOVA: Analysis of variance; APTT: Activated partial thromboplastin time; AST: Aspartate aminotransferase; D-D: D-dimer; DNA: Deoxyribonucleic acid; HBsAg: Hepatitis B surface antigen; HBV: Hepatitis B virus; ICU: Intensive care unit; IPM: Intermittent Pringle maneuver; PM: Pringle maneuver; PT: Prothrombin time; RCT: Randomized controlled trial; TNM: Tumor, node, metastasis

\section{Acknowledgements}

Not applicable.

\section{Authors' contributions}

Study concept and design was done by XW. Literature research was done by WZ. Clinical studies were done by XW and XL. Data acquisition was done by TT. Data analysis was done by ZY and HL. Statistical analysis was done by ZY and WZ. Manuscript preparation was done by XW and XL. Manuscript editing was done by XW and XL. Manuscript review was done by XW and $\mathrm{XL}$. All authors read and approved the final manuscript.

\section{Funding}

None.

Availability of data and materials

All data generated or analyzed during this study are included in this published article.

\section{Ethics approval and consent to participate}

This study was performed in accordance with the Declaration of Helsinki and was approved by the Ethics Committee of Southwest Hospital, Third Military Medical University (Army Medical University).

\section{Consent for publication}

All included patients provided informed consent.

\section{Competing interests}

The authors declare that they have no competing interests.

Received: 4 July 2019 Accepted: 29 July 2019

Published online: 13 August 2019

\section{References}

1. Chen K, Qiu F, Tian Y, Lai Z, Zhou S, Yan M, et al. Application of hepatectomy without hepatic blood flow occlusion in treatment of primary liver cancer. J Clin Hepatol. 2014;30:1060-3.

2. Piardi $T$, Lhuaire M, Memeo R, Pessaux P, Kianmanesh R, Sommacale D. Laparoscopic Pringle maneuver: how we do it? Hepatobiliary Surg Nutr. 2016:5:345-9.

3. Smyrniotis V, Kostopanagiotou G, Lolis E, Theodoraki K, Farantos C, Andreadou l, et al. Effects of hepatovenous back flow on ischemicreperfusion injuries in liver resections with the pringle maneuver. J Am Coll Surg. 2003;197:949-54.

4. Fu SY, Lau WY, Li GG, Tang QH, Li AJ, Pan ZY, et al. A prospective randomized controlled trial to compare Pringle maneuver, hemihepatic vascular inflow occlusion, and main portal vein inflow occlusion in partial hepatectomy. Am J Surg. 2011;201:62-9.

5. Chen L, Luo Z, Fu W, Liao X, Cui Z, Zhou J. Detection of urinary metabolomics before and after Pringle maneuver-induced liver ischemia and reperfusion injury in rats using gas chromatography-mass spectrometry. Dis Markers. 2013;35:345-51.

6. Miyagi S, Nakanishi C, Kawagishi N, Kamei T, Satomi S, Ohuchi N. Pure laparoscopic hepatectomy combined with a pure laparoscopic pringle maneuver in patients with severe cirrhosis. Case Rep Gastroenterol. 2015;9: $101-5$. 
7. Man K, Fan ST, Ng IO, Lo CM, Liu CL, Yu WC, et al. Tolerance of the liver to intermittent pringle maneuver in hepatectomy for liver tumors. Arch Surg. 1999:134:533-9.

8. Ypsilantis P, Lambropoulou M, Tentes I, Anagnostopoulos K, Tsigalou C, Papadopoulos N, et al. Impaired liver regeneration following partial hepatectomy using the Pringle maneuver: Protective effect of mesna. J Gastroenterol Hepatol. 2009;24:623-32.

9. Wang PF, Li CH, Chen YW, Zhang AQ, Cai SW, Dong JH. Preserving hepatic artery flow during portal triad blood inflow occlusion improves remnant liver regeneration in rats after partial hepatectomy. J Surg Res. 2013;181: 329-36.

10. Yovita H, Djumhana A, Abdurachman SA, Saketi JR. Correlation between anthropometrics measurements, prealbumin level and transferin serum with Child-Pugh classification in evaluating nutritional status of liver cirrhosis patient. Acta Med Indones. 2004;36:197-201.

11. Liu A. Efficient Estimation of Two Seemingly Unrelated Regression Equations. J Multivar Anal. 2002:82:445-56.

12. Man K, Fan ST, Ng IO, Lo CM, Liu CL, Wong J. Prospective evaluation of Pringle maneuver in hepatectomy for liver tumors by a randomized study. Ann Surg. 1997;226:704-11 discussion 11-3.

13. Winbladh A, Bjornsson B, Trulsson L, Offenbartl K, Gullstrand P, Sandstrom P. Ischemic preconditioning prior to intermittent Pringle maneuver in liver resections. J Hepatobiliary Pancreat Sci. 2012;19:159-70.

14. Takatsuki M, Soyama A, Hidaka M, Kinoshita A, Adachi T, Kitasato A, et al. Prospective study of the safety and efficacy of intermittent inflow occlusion (Pringle maneuver) in living donor left hepatectomy. Hepatol Res. 2015;45: 856-62.

15. Park JB, Joh JW, Kim SJ, Kwon CH, Chun JM, Kim JM, et al. Effect of intermittent hepatic inflow occlusion with the Pringle maneuver during donor hepatectomy in adult living donor liver transplantation with right hemiliver grafts: a prospective, randomized controlled study. Liver Transpl. 2012:18:129-37.

16. Zhang $Y$, Yang $H$, Deng $X$, Chen $Y$, Zhu S, Kai C. Intermittent Pringle maneuver versus continuous hemihepatic vascular inflow occlusion using extra-glissonian approach in laparoscopic liver resection. Surg Endosc. 2016:30:961-70

17. Wang CC, Yap AQ, Chen CL, Concejero AM, Lin YH. Comparison of major hepatectomy performed under intermittent Pringle maneuver versus continuous Pringle maneuver coupled with in situ hypothermic perfusion. World J Surg. 2011;35:842-9.

18. van den Broek MA, Bloemen JG, Dello SA, van de Poll MC, Olde Damink SW, Dejong $\mathrm{CH}$. Randomized controlled trial analyzing the effect of 15 or 30 min intermittent Pringle maneuver on hepatocellular damage during liver surgery. J Hepatol. 2011:55:337-45.

19. Xia F, Lau WY, Qian C, Wang S, Ma K, Bie P. Surgical treatment of giant liver hemangiomas: enucleation with continuous occlusion of hepatic artery proper and intermittent Pringle maneuver. World J Surg. 2010;34:2162-7.

20. Torzilli G, Procopio F, Donadon M, Del Fabbro D, Cimino M, Montorsi M. Safety of intermittent Pringle maneuver cumulative time exceeding 120 minutes in liver resection: a further step in favor of the "radical but conservative" policy. Ann Surg. 2012;255:270-80.

21. van Riel WG, van Golen RF, Reiniers MJ, Heger M, van Gulik TM. How much ischemia can the liver tolerate during resection? Hepatobiliary Surg Nutr. 2016;5:58-71.

22. Lee KF, Wong J, Cheung SYS, Chong CCN, Hui JWY, Leung VYF, et al. Does Intermittent Pringle Maneuver Increase Postoperative Complications After Hepatectomy for Hepatocellular Carcinoma? A Randomized Controlled Trial. World J Surg. 2018;42:3302-11.

23. Hester CA, El Mokdad A, Mansour JC, Porembka MR, Yopp AC, Zeh HJ, et al. Current Pattern of Use and Impact of Pringle Maneuver in Liver Resections in the United States. J Surg Res. 2019;239:253-60.

24. Hao S, Yang X, Yang W, Wan C. Intermittent porta hepatis occlusion is a risk factor of postoperative intra-hepatic recurrence of hepatocellular carcinoma. Chin J Hepatobiliary Surg. 2013;19:321-4.

25. Huang J, Tang W, Hernandez-Alejandro R, Bertens KA, Wu H, Liao M, et al. Intermittent hepatic inflow occlusion during partial hepatectomy for hepatocellular carcinoma does not shorten overall survival or increase the likelihood of tumor recurrence. Medicine (Baltimore). 2014;93:e288.

26. Ferrero A, Russolillo N, Vigano L, Lo Tesoriere R, Muratore A, Capussotti L. Does Pringle maneuver affect survival in patients with colorectal liver metastases? World J Surg. 2010;34:2418-25.

\section{Publisher's Note}

Springer Nature remains neutral with regard to jurisdictional claims in published maps and institutional affiliations.

\section{Ready to submit your research? Choose BMC and benefit from:}

- fast, convenient online submission

- thorough peer review by experienced researchers in your field

- rapid publication on acceptance

- support for research data, including large and complex data types

- gold Open Access which fosters wider collaboration and increased citations

- maximum visibility for your research: over $100 \mathrm{M}$ website views per year

At BMC, research is always in progress.

Learn more biomedcentral.com/submissions 